BULL. AUSTRAL. MATH. SOC.

VOL. $30(1984), 169-173$.

\title{
A NOTE ON THE CENTRAL LIMIT THEOREM FOR GEODESIC RANDOM WALKS
}

\author{
GilLes BLUm
}

In this article we use a theorem of T.G. Kurtz to prove a central limit theorem for geodesic random walks.

\section{0 . Introduction}

In [1] Jorgensen defines the concept of a random walk in a general Riemannian manifold and investigates the behavior in the limit of a sequence of such random walks. His approach to the problem in question is based upon the use of semigroup methods due to Trotter and Stone. The aim of this note is to give a new proof of some of Jorgensen's results using instead the following theorem of Kurtz and several ideas in [2].

THEOREM 0.1. For $N=1,2, \ldots$ let $T_{N}$ be a linear contraction on a Banach space $L_{N}$, let $\varepsilon_{N}$ be a positive number and put $A_{N}=\left(T_{N}-I\right) / \varepsilon_{N}$. Assume that $\lim _{N \rightarrow \infty} \varepsilon_{N}=0$. Let $\{T(t)\}$ be a strongly continuous contraction semigroup on a Banach space $L$ with generator $A$ and let $D$ be a core for $A$. Let $\pi_{N}: L \rightarrow L_{N}$ be bounded linear operators such that $\sup _{N}\left\|\pi_{N}\right\|<\infty$. Then the following are equivalent:

(a) for each $f \in L, \quad\left\|T_{N}^{\left[t / \varepsilon_{N}\right]} \pi_{N} f-\pi_{N} T(t) f\right\| \rightarrow 0$ for alz

Received 13 March 1984.

Copyright Clearance Centre, Inc. Serial-fee code: 0004-9727/84 $\$ A 2.00+0.00$. 
$t \geq 0$;

(b) for each $f \in D$ there exists $f_{N} \in L_{N}$ such that $\left\|f_{N}-\pi_{N} f\right\| \rightarrow 0$ and $\left\|A_{N} f_{N}^{-\pi} A f\right\| \rightarrow 0$.

\section{A limit theorem}

Let $M$ be a Riemann manifold of dimension $n$. The Brownian motion in $M$ is a stochastic process $\left\{X_{t}^{x}: t \geq 0\right\}$ with continuous sample paths such that $x_{0}^{x}=x$ and

$$
f\left(x_{t}^{x}\right)-f(x)+\int_{0}^{t} \frac{a}{2}(\Delta f)\left(X_{s}^{x}\right) d s, a>0 \text {, }
$$

is a martingale for every $f \in C_{0}^{\infty}(M)$. (See [2]).

Let $x \in M, T_{x}(M)$ be the tangent space of $M$ at $x, S_{x}(i v)$ the unit sphere of $T_{x}(M), S(M)$ the bundle of tangent unit spheres. Let $\xi_{x} \in S_{x}(M)$ and $x\left(\cdot, \xi_{x}\right)$ be the unit speed geodesic starting from $x$ in the direction $\xi_{x}$. Let $\{r(k): k=0,1, \ldots\}$ be identically independantly distributed in $\mathbb{R}^{+}$with distribution $P$. Assume $E(\tau(k))=1, E\left(\tau^{2}(k)\right)=a, E\left(\tau^{3}(k)\right)<\infty$. Let $\{z(k): k=0,1, \ldots\}$ be the random variables in $S(M)$ such that

$$
z(0)=\left(x_{0}, \xi_{0}\right), \ldots, z(k)=\left(x_{k}, \xi_{k}\right)
$$

where

$$
\begin{aligned}
& x_{k}=x_{k-1}\left(\tau(k-1), \xi_{k-1}\right), \\
& \xi_{k}=\xi_{x_{k}},
\end{aligned}
$$

and the conditional distribution of $\xi_{k}$ given $\left\{\left(x_{0}, \xi_{0}\right), \ldots,\left(x_{k-1}, \xi_{k-1}\right)\right\}$ is the uniform distribution on $s_{x_{k}}$ denoted by $\mu$. The sequence $\left\{x_{0}, x_{1}, \ldots\right\}$ is called a geodesic random walk with identically distributed steps. (See [1] and [2].) 
THEOREM 1.1. For $N=1,2, \ldots$ let $x_{0}^{N}=x$ and

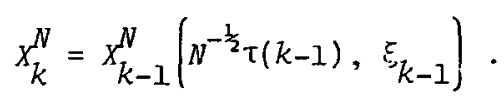

Assume the Ricci curvature of $M$ is bounded from below. Then for every $f$ in $C(M), \lim _{N \rightarrow \infty} E\left[f\left(X_{[N t]}^{N}\right]\right]=E\left[f\left(X_{t / n}^{x}\right)\right]$.

Proof. In the notations of Theorem 0.1 , let $L_{N}=B(S(M))$, $D=C_{K}^{2}(M)$. Let $P \cdot(x, \xi)$ be the parallel transport along the geodesic $X(\cdot, \xi)$. For $N=1,2, \ldots$ let $\left\{z_{N}(k)=\left(x_{k}^{N}, \xi_{k}\right): k=0,1, \ldots\right\}$ and for $f \in L_{N}$ define

$$
\begin{aligned}
T_{N} f(x, \xi) & =\iint f\left(x\left(\tau_{1} N^{-\frac{1}{2}}, \xi\right), P_{N^{-\frac{1}{2}} \tau_{1}}(x, \xi)(n)\right) d P \mu(d \eta), \\
A_{N} & =N\left(T_{N}-I\right) .
\end{aligned}
$$

(Note that $T_{N}$ is the linear contraction associated to the Markov chain $Z_{N}$ in $S(M)$.)

$$
\begin{aligned}
\text { For } f \text { in } & c_{K}^{2}(S(M)), \xi \in S_{x}(M), \eta \in S_{x}(M) \text { let } \\
& u(t, x, \xi, \eta)=f\left(X(t, \xi), P_{t}(x, \xi)(n)\right) .
\end{aligned}
$$

Then clearly

$$
\frac{\partial u}{\partial t}(0, x, \xi, \eta)=\xi^{i} \frac{\partial u}{\partial x_{i}}-\Gamma_{j, k^{i}}^{j} \eta^{j} \frac{\partial u}{\partial \xi_{i}} .
$$

Let $f \in D, \quad h=D_{\xi} f, k=(a / 2) D_{\xi \xi} f, f_{N}=f+N^{-\frac{1}{2}} h+N^{-1} k$ and $A=-(a / 2 n) \Delta f$. We show that $\left\|A_{N} f_{N}-A f\right\| \rightarrow 0$ as $N \rightarrow \infty$. Using (1.1) we have the following Taylor expansions for $f$ and $h$ (we replace $\tau(1)$ by $\tau_{1}$ and denote $\partial u / \partial t$ by $D_{\xi}^{n} f$ and $D_{\xi}^{\xi}$ by $\left.D_{\xi}\right)$, 


$$
\left|f\left(x\left(N^{-\frac{1}{2}} \tau_{1}\right)\right)-f(x)-N^{-\frac{1}{2}} \tau_{1} D_{\xi} f(x)-N^{-1} \tau_{1}^{2} D_{\xi} D_{\xi} f(x)\right|<K N^{-3 / 2} \tau_{1}^{3},
$$

(1.3) $\left|h\left(x\left(N^{-\frac{1}{2}} \tau_{1}, \xi\right), P_{N^{-\frac{3}{2}} \tau_{1}}(x, \xi)(\eta)\right)-h(x, \eta)-N^{-\frac{1}{2} \tau_{1}} D_{\xi}^{\eta} f(x, \eta)\right|$

For $g \in C(S(M))$ let $B$ be defined by

$$
B g(x, \xi)=\int_{S_{x}(M)} g(x, \eta) \mu(d n)-g(x, \xi) .
$$

By $(1.2),(1.3)$ and the definition of $T_{N}$ there exists a constant $K$ depending on $M$ only such that

$$
\left.N\left|T_{N} f(x, \xi)-f(x)-N^{-\frac{1}{2}} D_{\xi} f(x)-(\alpha / 2) N^{-1} D_{\xi} D_{\xi} f(x)\right| \leq N^{-\frac{1}{2}} K E\left(\tau^{3}\right]\right),
$$

(1.6) $\left.\quad \bar{N}\right|_{T} h(x, \xi)-h(x, \xi)-B h(x, \xi)-N^{-\frac{1}{2}} \int \eta_{\xi}^{\eta} h(x, \eta) \mu(d \eta) \mid \leq N^{-\frac{1}{2}} \alpha K$,

$$
\left|T_{N} k(x, \xi)-k(x, \xi)-B k(x, \xi)\right|<N^{-\frac{1}{2}}(a / 2) K .
$$

Using then a triangle inequality

$$
\begin{aligned}
\left|A_{N} f_{N}(x, \xi)-A f(x)\right| \leq N^{-\frac{1}{2}} & \left\{\left(E\left|\tau_{1}^{3}\right|+(3 / 2) a\right)+\left|B h(x, \xi)+D_{\xi} f(x)\right| N^{\frac{1}{2}}\right. \\
& +\left|B k(x, \xi)+(a / 2) D_{\xi \xi} f(x)+\int D_{\xi}^{\eta} f(x, n) \xi(d n)-A f(x)\right| .
\end{aligned}
$$

To conclude the proof we have to show that

$$
\begin{gathered}
B h(x, \xi)+D_{\xi} f=0, \\
\int D_{\xi}^{\eta} h(x, \eta) \xi(d \eta)=0,
\end{gathered}
$$

Since $\int n^{i} \xi\left(d n^{i}\right)=0$,

$$
B k(x, \xi)=A f(x)-(a / 2) D_{\xi} D_{\xi} f(x)
$$

$$
B h(x, \xi)_{i}=\int \eta^{i} \frac{\partial f}{\partial x_{i}} \xi\left(\partial n^{i}\right)-\xi^{i} \frac{\partial f}{\partial x_{i}}=-\xi^{i} \frac{\partial f}{\partial x_{i}},
$$

and (1.8) follows. (1.9) follows from the fact that 


$$
\begin{aligned}
& \text { Central limit theorem } \\
& D_{\xi}^{\eta_{h}} h(x, \eta)=\xi^{i} \eta^{k} \frac{\partial^{2} f}{\partial x_{i} \partial x_{k}}-\Gamma_{j, k}^{i} \xi^{j} \eta^{k} \frac{\partial f}{\partial x_{k}}
\end{aligned}
$$

and

$$
\int D_{\xi}^{\eta} h(x, \eta) \xi(d n)=\left(\xi^{i} \frac{\partial^{2} f}{\partial x_{i} \partial x_{k}}(x)-\Gamma_{j, k}^{k} \xi^{j} \frac{\partial f}{\partial x_{k}}\right) \int \eta^{k} \mu\left(d \eta^{k}\right)=0 .
$$

For (1.10) see [2], p. 209. Since clearly $\left\|f_{N}-f\right\| \rightarrow 0$ Theorem 0.1 (a) is satisfied and Theorem 1.I follows easily.

\section{References}

[1] Erik Jorgensen, "The central limit problem for geodesic random walks", 2. Wahrsch. Verw. Gebiete 32. (1975), 1-64.

[2] Mark Pinsky, "Stochastic Riemannian geometry", Probabilistic analysis and related topics, 199-236 (Academic Press, New York and London, 1978).

[3] T.G. Kurtz, "Extensions of Trotter's operator semigroup approximation theorems", J. Funct. Anal. 3 (1969), 111-132.

Department of Mathematics,

Case Western Reserve University,

Cleveland,

Oh io 44106 ,

USA. 